\title{
On the tension between Large Scale Structures and Cosmic Microwave Background
}

\author{
Marian Douspis* \\ Institut d'Astrophysique Spatiale, CNRS, Université Paris Sud, Orsay, France \\ E-mail: marian.douspiseias.u-psud.fr
}

\section{Laura Salvati}

Institut d'Astrophysique Spatiale, CNRS, Université Paris Sud, Orsay, France

E-mail: laura.salvati@ias.u-psud.fr

\section{Nabila Aghanim}

Institut d'Astrophysique Spatiale, CNRS, université Paris Sud, Orsay, France

E-mail: nabila.aghanimeias.u-psud.fr

Recent years have brought strong observational evidences for the standard LCDM cosmological model. Cosmic microwave background (CMB) anisotropy and large scale structure (LSS) probes do not favour any extensions of the standard model. Nevertheless, in this framework, the prefered cosmological parameters may differ from probe to probe, from experiment to experiment. This is the well known case of the tension between CMB and Sunyaev Zel'dovich (SZ) galaxy clusters (GC) from Planck $\dagger$ In 2013, the Planck team has shown that the prefered matter content $\left(\Omega_{M}\right)$ and density fluctuation power spectrum amplitude $\left(\sigma_{8}\right)$, the two main cosmological parameters probed by the galaxy cluster number count, are different in the CMB analyses and in the SZ cluster analyses at more than 2 sigmas (a result confirmed in subsequent analyses). We present the results of our new analysis using more recent measurements of the CMB, SZ clusters and SZ power spectrum of 2016 and show that the tension on $\left(\Omega_{M}, \sigma_{8}\right)$ is mostly releaved. The lower value of the reionisation optical depth and thus of $\sigma_{8}$ in the recent Planck studies is the main reason. We also show that basic extensions of the standard model (massive neutrinos or nonlambda dark energy) do not help improving the agreement between the probes. In order to fully reconcile SZ clusters with CMB best model, the mass of the galaxy clusters should be $40 \%$ lower than derived from hydrostatic equilibrium estimates. While current numerical simulations and weak lensing measurements agree for a mass bias of $20 \%$, investigations are still going on to explain such disagreement on the mass bias. We show that considering a mass bias evolving with redshift or mass does not help in eliminating the discrepancy.

2nd World Summit: Exploring the Dark Side of the Universe

25-29 June, 2018

University of Antilles, Pointe-à-Pitre, Guadeloupe, France

\footnotetext{
${ }^{*}$ Speaker.

${ }^{\dagger}$ planck.esa.int
} 


\section{A cosmological tension?}

Galaxy clusters are thought to be strong probes of the cosmological model because of their large scales, dark matter dominated content and thus sensitivity to the global evolution of the Universe rather than baryonic micro-physics. Samples of GC have been used in several frequency domains (optical, X-ray, SZ) to put constrains on cosmological parameters. Only recently, samples were large enough to make results almost insensitive to statistical errors. This has been the case in SZ, when Planck published it first catalogue [4] and used a subsample of circa 200 clusters [1] to probe the standard cosmological model. Constraints form GC were shown to be 2.4- $\sigma$ away from the CMB (Fig. 1, right). In parallel, in the last years, galaxy surveys (e.g. KIDS, CFHTLS, DES) have produced weak lensing constraints on the same parameters showing as well some tension with CMB results. While all experiments contain systematics that may explain the small shift in prefered cosmological parameters, it is puzzling that all low redshift large scale structure probes are in agreement and drive $\sigma_{8}$ towards lower values than the CMB (Fig. 1, left).
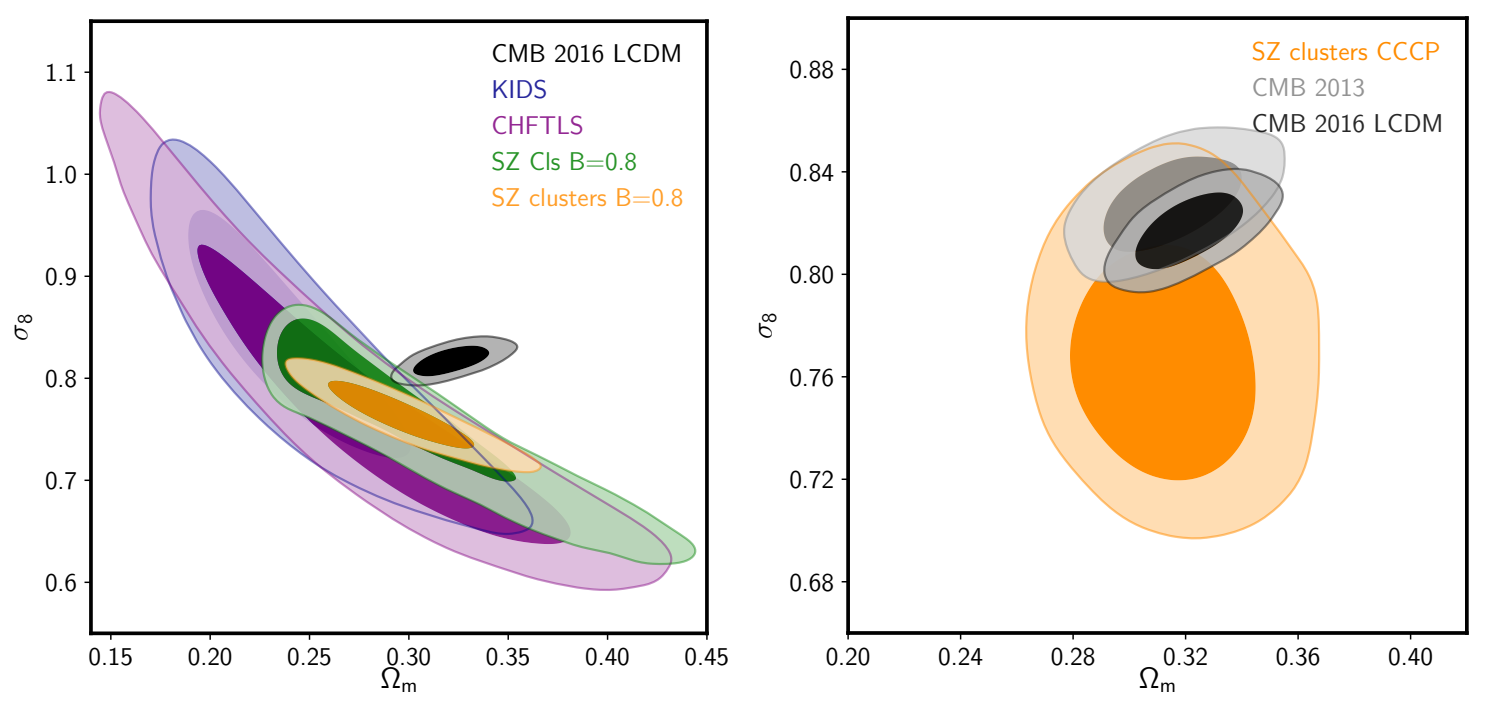

Figure 1: Left: recent large scale constraints compared with CMB (black filled contours). See text for references. Right: Comparison of SZ cluster constraints from Planck (yellow) with CCCP prior on the mass bias and Planck CMB constraints from 2013 and 2016 (grey and black respectively). The displacement of the CMB constraints is due to the new estimate of the reionisation optical depth. Adapted from [3].

In [3] we have performed a new analysis of the SZ signal from Planck, combining the number counts in redshift and signal-to-noise ratio (NC), of circa $400 \mathrm{SZ}$ clusters [2], with the SZ power spectrum (CL) derived from 50\% of the MILCA SZ map [5, 6]. We compared and combined the derived constraints with the $\mathrm{CMB}$ assuming the new value of the optical depth of reionisation $\tau=0.055 \pm 0.009$ from Planck [7].

We show in Fig. 1 (right) that the tension is reduced to $1.5 \sigma$ with such a new value of $\tau$. A full agreement between the two probes is not yet completely achieved when assuming the default value of the mass bias, $(1-b) \sim 0.8$ (CCCP prior [8], see next section), and new tests on extensions of the standard cosmological model do not help (Fig. 2, left). In the three cosmological models we probe (LCDM, massive neutrinos, dark energy), the prefered value of the mass bias is always much 
lower than the default value $(\sim 0.8)$ as shown in Fig. 2 (right). A value of $(1-b) \sim 0.62$ (a 38\% lower hydrostatic estimate compared to the true mass) is needed to reconcile CMB and SZ probes. Furthermore, such a value leads to a baryon fraction in clusters at odd with the universal value [9].
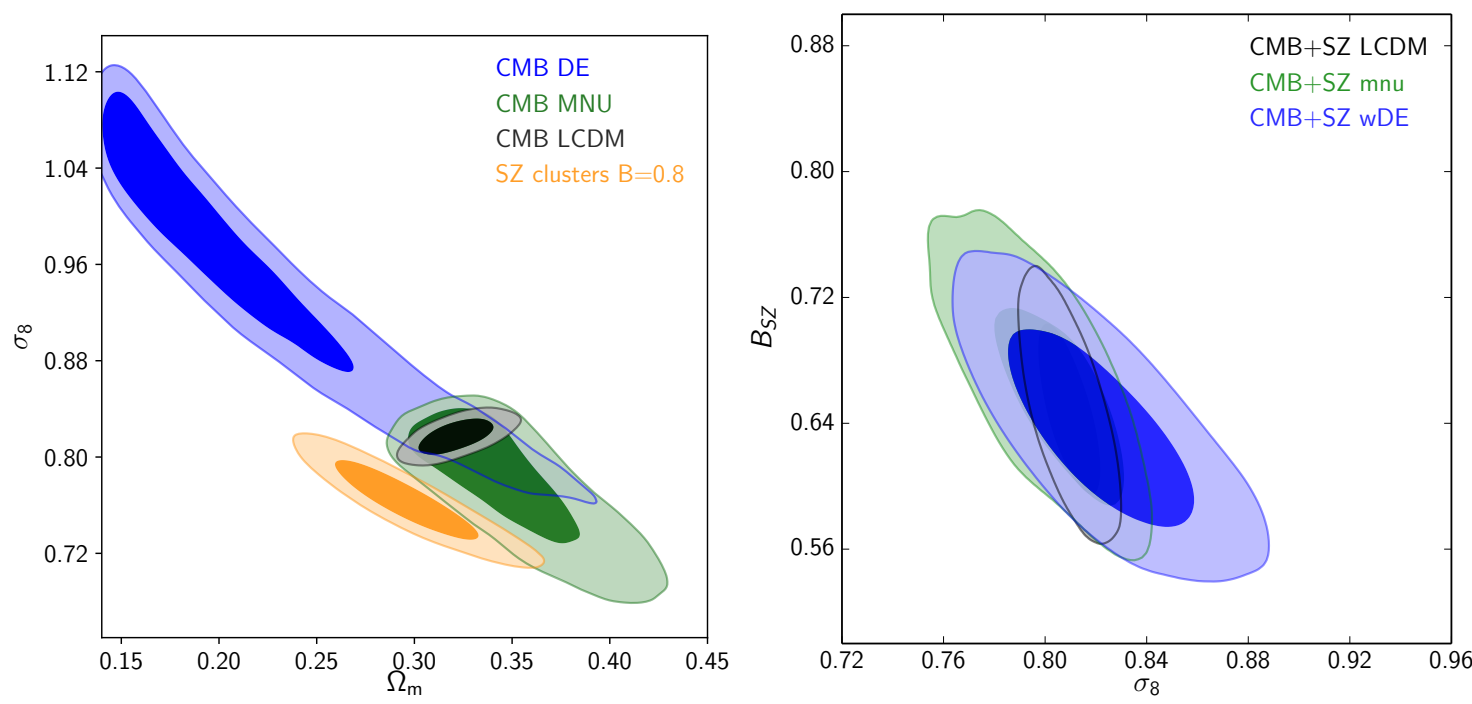

Figure 2: Left: Comparison of cosmological paramater when opening up the extensions of LCDM (massive neutrinos (green) and dark energy (blue)) for CMB. LCDM contours are in black. Right: Constraints on the mass bias $\left(B_{S Z}\right)$ and $\sigma_{8}$ for the different cosmological model extensions when combining CMB and SZ.

\section{An astrophysical tension?}

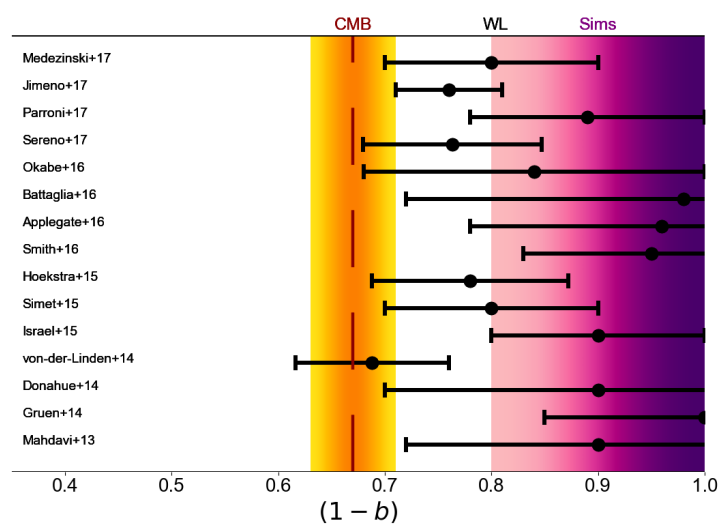

Figure 3: Mass bias estimates from simulations (purple dashed area) and lensing estimates from different teams (black points with errors), compared the value needed for reconciling CMB and SZ (orange area).

The main systematic of the SZ cluster analyses is the mass bias: the ratio between the hydrostatic mass estimates (HME) and the true mass. The HME are derived from X-ray and SZ data [4]. The true mass is not an observable and can be assumed to be derived from numerical simulations 
(NS) or lensing measurements (lensing mass estimates, LME). In [1] the bias (1-b) is computed by comparing the observed mass (from Xray and SZ) with a set of 12 numerical simulations (see citations therein). In the following studies, a prior on the bias coming from HME and LME comparison is usually assumed. Figure 3 summarizes the recent values of the bias derived from both NS and LME. As stated above, the low value of $(1-b)$ needed to retrieve CMB cosmology from clusters is marginally consistent with other estimates. A unique constant value of the mass bias cannot thus explain the remaining tension between CMB and SZ.
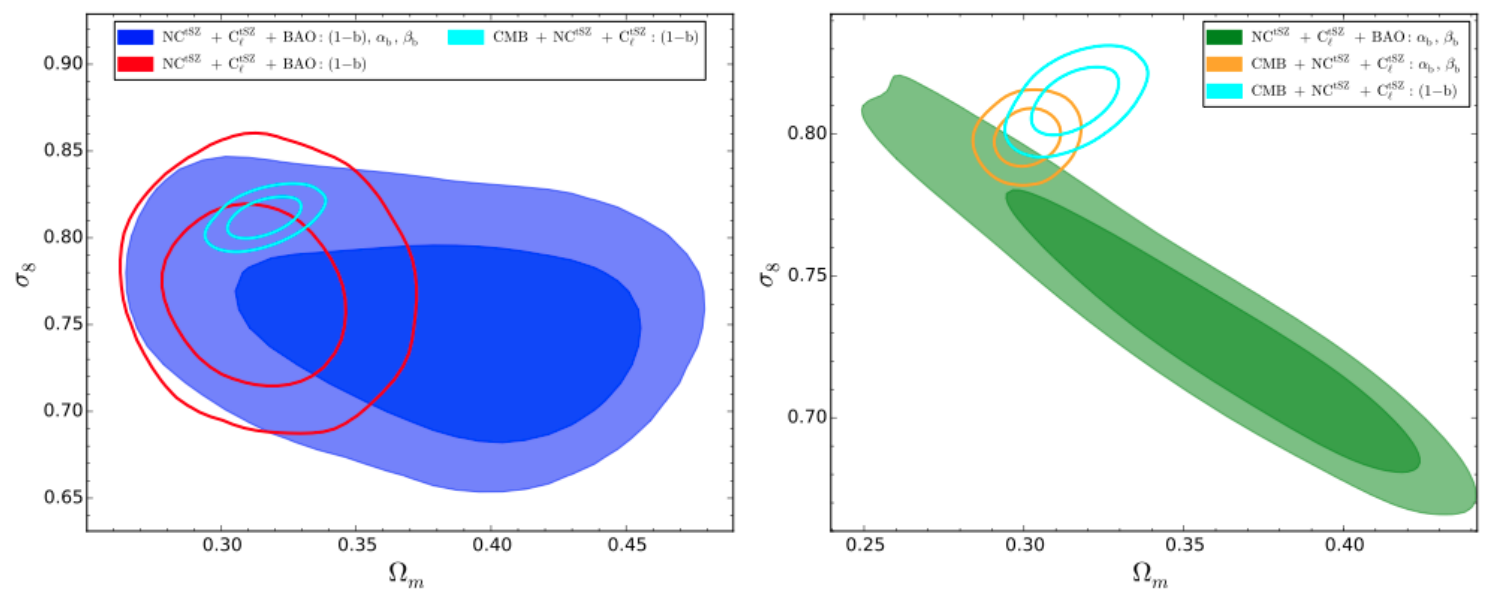

Figure 4: Constraints from different test of SZ and CMB probes. Left: default constraints from SZ (red) and CMB (cyan) are compared with SZ constraints with evolving bias free (blue). Right: the light green area shows the SZ constraints assuming a fixed amplitude of the bias to 0.75 but letting the mass and redshift indeces free. The yellow contours is given by the combination of the latter with $\mathrm{CMB}$, and offers a really bad goodness of fit of both CMB and SZ data. Adapted from [10].

In order to investigate further the solutions to reconcile the 2 probes, we study the possibility that the mass bias is in fact depending on the mass and redshift of the clusters. If the HME is not a good proxy for the total mass, it may be due to baryonic effects, non thermal pressure, magnetic field, ...; processes that may be non universal and linear, and vary with the mass and redshift of the clusters. We thus consider the following evolution of the mass bias [10]:

$$
(1-b)_{\mathrm{var}}=(1-b) \cdot\left(\frac{M}{M_{*}}\right)^{\alpha} \cdot\left(\frac{1+z}{1+z_{*}}\right)^{\beta}
$$

where $(1-b)$ is an amplitude at a given mass and redshift, $M_{*}=6 \cdot 10^{14} M_{\odot}$ to be consistent with the pivot mass of the scaling relations (see Eqs. (7) and (8) in [2]) and $z_{*}=0.22$ is the median value of the clusters catalog that we are considering. When all bias parameters $(1-b, \alpha, \beta)$ are let free in addition to the cosmological parameters, the contraints of SZ obviously weakens but are not in better agreement with CMB ones as shown in Fig. 4 (left). In addition, we don't find evidence for any evolution of the bias with mass and redshift at more than $1 \sigma$. We reach the same conclusions when combining CMB and SZ probes. Furthermore, when the mass bias is fixed to an optimistic value of $(1-b)=0.75$ at the mean mass and redshift of the cluster sample, allowing for redshift and mass dependencies does not drive the cluster constraints towards the CMB ones. Figure 4 (right) shows the contours of SZ alone with such a fixed bias (green), compared to CMB 
(cyan). The yellow constraints are obtained when combining CMB and SZ with fixed $(1-b)$ and free mass and resdshift indeces. The goodness of fit of such combination is very bad, showing that no reasonable evolving bias exists to reconcile $\mathrm{CMB}$ and $\mathrm{SZ}$ probes.

\section{Conclusions}

The tension between the cosmological models prefered by CMB and SZ data is reduced thanks mostly to the new value of the optical depth of reionisation observed by Planck since 2016, but both probes lead to differents views on the mass estimates of clusters. Simple dark energy models (constant equation of state) and massive neutrinos are not appropriate extensions to reduce fully the remaining tension. Modified gravity scenari may provide a good cosmological model allowing to satisfy both probes, but in simple scenarios, extreme departure from general relativity are needed [12]. In the LCDM model, the "simple" way to reconcile both CMB and SZ is to require a high mass bias (low value of $(1-b) \sim 0.6$ ), implying $40 \%$ missing mass when estimated through hydrostatic equilibrium. Such a low value is not supported by numerical simulations nor lensing mass measurements. When this bias is allowed to be evolving with redshift and mass, the cluster constraints weakens but do not reach the CMB ones. Furthermore, no evidence for varying bias is found. Being it constant or evolving, no value of the bias is found to satisfy for SZ clusters and CMB observations.

\section{References}

[1] Planck Collaboration, Ade, P. A. R., Aghanim, N., et al. 2014, A\&A, 571, A20

[2] Planck Collaboration, Ade, P. A. R., Aghanim, N., et al. 2016, A\&A, 594, A24

[3] Salvati, L., Douspis, M., \& Aghanim, N. 2018, A\&A, 614, A13

[4] Planck Collaboration, Ade, P. A. R., Aghanim, N., et al. 2014, A\&A, 571, A29

[5] Hurier, G., Macías-Pérez, J. F., \& Hildebrandt, S. 2013, A\&A, 558, A118

[6] Planck Collaboration, Aghanim, N., Arnaud, M., et al. 2016, A\&A, 594, A22

[7] Planck Collaboration, Adam, R., Aghanim, N., et al. 2016, A\&A, 596, A108

[8] Hoekstra, H., Herbonnet, R., Muzzin, A., et al. 2015, MNRAS, 449, 685

[9] Eckert, D., Ghirardini, V., Ettori, S., et al. 2018, arXiv:1805.00034

[10] Salvati, L., Douspis, M., \& Aghanim, N. 2018, in prep.

[11] Planck Collaboration, Aghanim, N., Akrami, Y., et al. 2018, arXiv:1807.06209

[12] Sakr, Z., Ilić, S., \& Blanchard, A. 2018, arXiv:1803.11170

\section{Acknowledgments}

Based on observations obtained with Planck (http://www.esa.int/Planck), an ESA science mission with instruments and contributions directly funded by ESA Member States, NASA, and Canada. Part of this research has been supported by the funding for the ByoPiC project from the European Research Council (ERC) under the European Union's Horizon 2020 research and innovation programme grant agreement ERC-2015-AdG 695561. 